\title{
Online Shopping Featuring "My Customized Avatar" - Generating Customized Avatars for a Sustainable Shopping Experience in E-commerce
}

\author{
Michael ERNST *, Antje CHRISTOPHERSEN *, Silke SANDULOFF * \\ Niederrhein University of Applied Sciences, Germany \\ https://doi.org/10.15221/21.34
}

\begin{abstract}
Nowadays E-commerce and E-shopping become more and more popular, supported by the actual pandemic situation on one side but especially driven by technology and society transformation on the other. The product development process in clothing industry is in a state of constant change, implementing more and more digital, and especially virtual tools, within the past years. [1] Beside the product itself and physical as well as optical virtual fabrics and trimmings, especially the avatar plays an important role in this process - from the beginning in design and ending up in Ecommerce when presenting the products on matching avatars gains an attractive importance for the consumer.

Companies developing their collections and products for target groups are aiming for fit and brand consistency.

Anyway, within the product development process, there is a totally different understanding when it comes to design of avatars, visual expression on one side and technical and measurement reliable avatars on the other. [1,2]

Beside the question of individualization of customized avatars, the importance of avatar design at the beginning of the process becomes "cruelly" important again at the end when getting in "touch" with the customer and its individual and subjective feelings. [3,4]

Though the complete product development process is lifted up to a cloud-based system - at the end of the day a valuable customer has to buy the products to make them "alive", and keep the company running.
\end{abstract}

Keywords: 3D body scan; avatar; sizing and fit, 3D simulation, virtual product; development, rendering, E-commerce

\section{Introduction}

Within the product development process in clothing industry the introduction of 3D CAD technology for clothing simulation has directly made a fuss about the avatar design. Still there is a gap between the ideal avatar from a design point of view compared to the pattern department and the technical department responsible for fit control. This indifference is also given again when it comes to Ecommerce and E-shopping platforms where customer can check their individual fit on given adjusted avatars. The question arises if customers really want to see a perfect twin of themselves during a virtual shopping experience with all detailed measurements and expressions? Or if they prefer - similar to reality - to get a first impression on an avatar, an adaptive model only representing the main silhouette with few basic measurements.

On the other side it is important to know the limits of clothing simulation on modified avatars [5] - Is it worth to offer a huge test population for details in measurement variation if the common user cannot see really a difference in the simulation to made a decision for the product and the size? Also the visual effect assigned to the products by applying texture maps can easily convince a customer - a color reliability along the product development process assumed and given - but can also superimpose the fitting result and end up - together with misunderstanding the fabric touch and feeling - in high return rates. Thus "My customized avatar" has to been checked against various needs and restrictions and fulfill mainly customer demands for a successful E-shopping experience, leaving one's mark for longtime customer retention.

*michael.ernst@hs-niederrhein.de, +49 2161 186-6080;

*antje.christophersen@hs-niederrhein.de, ”silke.sanduloff@hs-niederrhein.de 


\section{Research Methodology}

Investigations have been carried out to build up a reliable process, starting from traditional 3D body scanning technology on one side but also based on an algorithm to define individual avatars from a two side body photo view, by applying an elliptical calculation and in a first approach from basic limited information such as body height and body weight, to offer a customized avatar along the E-shopping pipeline. One of the most important parts deals with the maintenance of measurement reliable avatar structures up to high-end visualization of fitted avatars by using rendering systems. Especially the challenge of what is acceptable and expectable by the consumer, in between objective and subjective definition and feelings, is unneglectable at all and in comparison, to design branding of 3D models used.

Therefore 12 different female and male bodies in four different ageing groups from 20 to 60 years old have been scanned and investigated in detail under different aspects to generate in a first approach basic "my customized avatars" - in line with measurements and posture requirements and fitted - after size prediction - with best fitted preselected clothing products: shirt, blouse, pants, dress, jacket- single and in combination as outfit with assigned physical fabric parameters. Apart from fit and design, the look of the avatar has been modified to investigate the acceptance of E-shoppers on one side and the environment settings on the other.

In a second approach the customer related avatar was completely exchanged by a virtual human being with all advantages and special features to highlight the overall impression of the presentation and to rate the benefit- additionally to features such as pose and animation, coming with. [Fig.1]

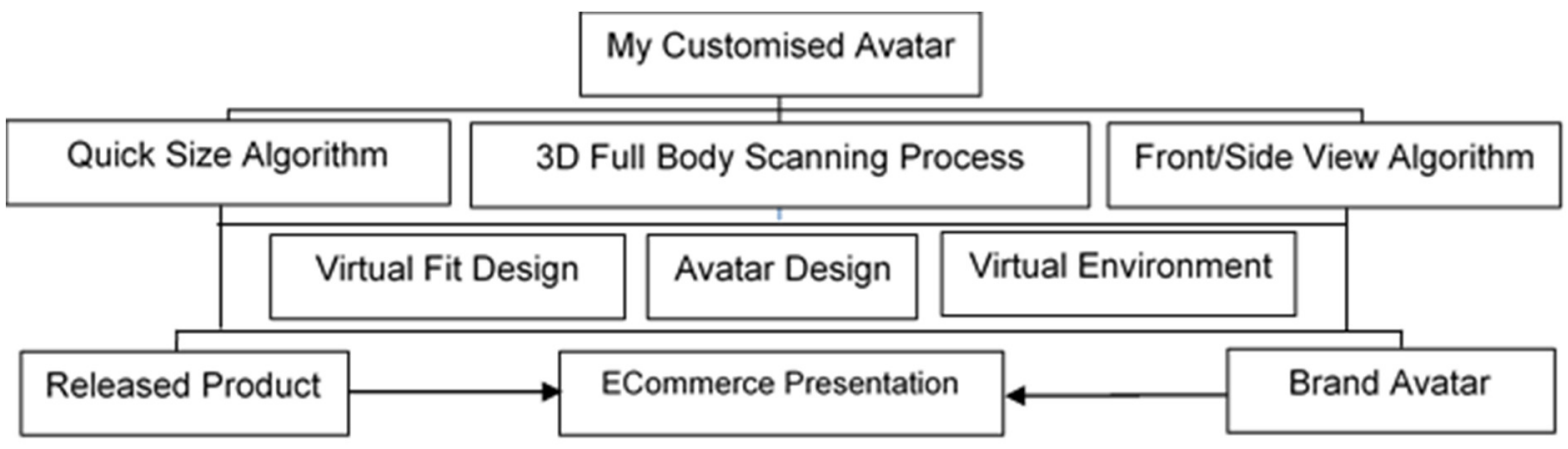

Figure 1: Research Methodology for "E-shopping- featuring my customized avatar"

\section{Body Data and Avatar Generation}

Body data and body measurements have been questioned and captured by investigating three main different approaches, to finally give a size prediction and offering an avatar to the customer for a virtual fitting experience.

Usually a 3D full body scanning service is not available at home. Anyway, this technology was taken for data comparison, data validation and representing the solution eventually given in a shop solution offering both - E-shopping and live experience.

Comparing the results of all these technologies results in body measurements strongly related to the algorithm taken and therefore can be widely distributed and overcoming easily the grading value assigned to them. Especially when it comes to individual bodies the location of the waist already can be quite tricky- with a direct impact to most of the height measurements and therefore changing the complete size set. Anyway, for mass production- not MtM made to measure or tailor made, only three measurements (bust, waist, hip) have been investigated in a first approach, fitted with additional measurements if the product is "asking" for. 


\subsection{Quick Size Algorithm}

Mostly people struggle when it comes to privacy and uploading selfies and critical personal data to a unknown E-commerce platform - especially with body shapes not representing the ideal fashion idea and in a certain ageing group.

The easiest approach in this case is to ask for at least age, body weight and body height.

By analyzing thousands of scans, taken in our labs over the past years, it is possible to generate in a first approach a so-called quick size function, combining the three mentioned parameters, resulting in a simple function for predicting body measurements, aligned with statistical distribution. Literally this simple approach cannot "catch" individual body shapes, away from the standard and the mean. Anyway this algorithm is the easiest way for size prediction and showing an avatar to a customer without asking for more information- e.g. needed honestly answered questions about body shape, bra size or ticking on little icons showing different silhouettes like apple shape or bottle neck shape.

In a first approach and referring to single parameters, this function can be simplified to linear functionscombining body weight with given body height and age. In fig. 2 , this relation is given for females in age group 4 (50-60 years), calculated for body height $168 \mathrm{~cm}$ with variation of body weight for bust (uBr), waist (uTa) and hip (uGe).

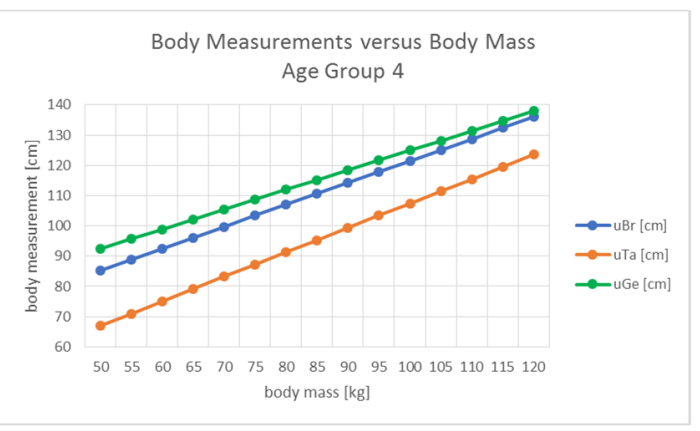

Figure 2: Virlab Quick Size Function $1^{\text {st }}$ approach; Age Group 4

Checking the process with three females the deviation shown in table 1 strongly depends on individual body shape not captured and represented by the quick size functions - only asking for age, body height and body weight. The more the body shape differs from the harmonized distribution of a scanned population the more the difference in body measurements of effected zones. Anyway, double checking the system by calculating the measurements for a common target population shows that more than $75 \%$ are in the limits of one grading step (usually $3-4 \mathrm{~cm}$ )

It is important to mention that the clothing is usually represented by ready measurement charts. The ease coming from construction, design and fabric analyzing and implementing these values for production pattern, all this is related to body measurements but is also effected strongly by brand and manufacturing process steps and difficult to control. Therefore, all the products in E-commerce especially when coming from different suppliers have to be checked and rated and assigned to target size prediction models respecting also - if available via $\mathrm{KI}$ algorithm, the individual shopping behavior of individual customers and additional information available.

The hand measurements from expert (HME) for bust, waist and hip given in table 1 have been taken by three experts independently one from each other, according to standard, to ensure the best accuracy by comparing these measurements and if necessary taking the average. Two values are overcoming the $4 \mathrm{~cm}$ grading limit: Female F2 shows a strong hip expression and female 3 shows a lower bust expression than given by the approximation of the QSF calculation. In a first approach this values will result in adapted size prediction - not knowing the individual fit estimation of the customer nor respecting the ready measurements of the clothing and the product description - e.g. dress with flared skirt- not affecting the critical hip measurement of female 2.

\begin{tabular}{|l|r|r|r|r|r|r|r|r|r|}
\hline Age Group 4 & \multicolumn{4}{|c|}{ Female F1 } & \multicolumn{3}{|c|}{ Female F2 } & \multicolumn{3}{|c|}{ Female F3 } \\
\hline body measurement [cm] & QSF & HME & Diff & QSF & HME & Diff & QSF & HME & Diff \\
\hline bust (uBr) & 107,8 & 106 & $-1,8$ & 111,5 & 109 & $-2,5$ & 97,5 & 93 & $-4,5$ \\
\hline waist (uTa) & 91,9 & 89 & $-2,9$ & 97,1 & 97 & $-0,1$ & 80,8 & 82 & 1,2 \\
\hline hip (uGe) & 113,4 & 116 & 2,6 & 112,7 & 117 & 4,3 & 103,1 & 102 & $-1,1$ \\
\hline
\end{tabular}

Table 1: Virlab Quick Size Function (QSF) ${ }^{\text {st }}$ approach, female age group 4, hand measurements expert (HME) 


\subsection{D Full Body Scanning Process}

3D full body scanning was carried out by scanning the subjects with four different body scanners available in our scanning lab:

- TG3D Scanatic 360 Body Scanner; Infrared Technology (I)

- Vitus Anthroscan, Avalution GmbH, Laser Scanning Technology (L)

- Ditus Smart; Avalution GmbH; Kinect Sensor Technology 12x Kinect (K12)

- StyKu; Styku; Kinect Sensor Technology 1x Kinect (K1), turntable

In Fig. 3 the TG3D Scanatic 360 Body Scanner is shown. Working with app connect the body measurements are available within seconds after the scan and can be investigated and adapted if necessary within $\mathrm{mtm}$ tg3ds and finally - after defining the armhole region and crotch area downloaded as avatar (obj.) or live avatar with built in skeleton (fbx) for importing into 3D software tools e.g. DCSuite.
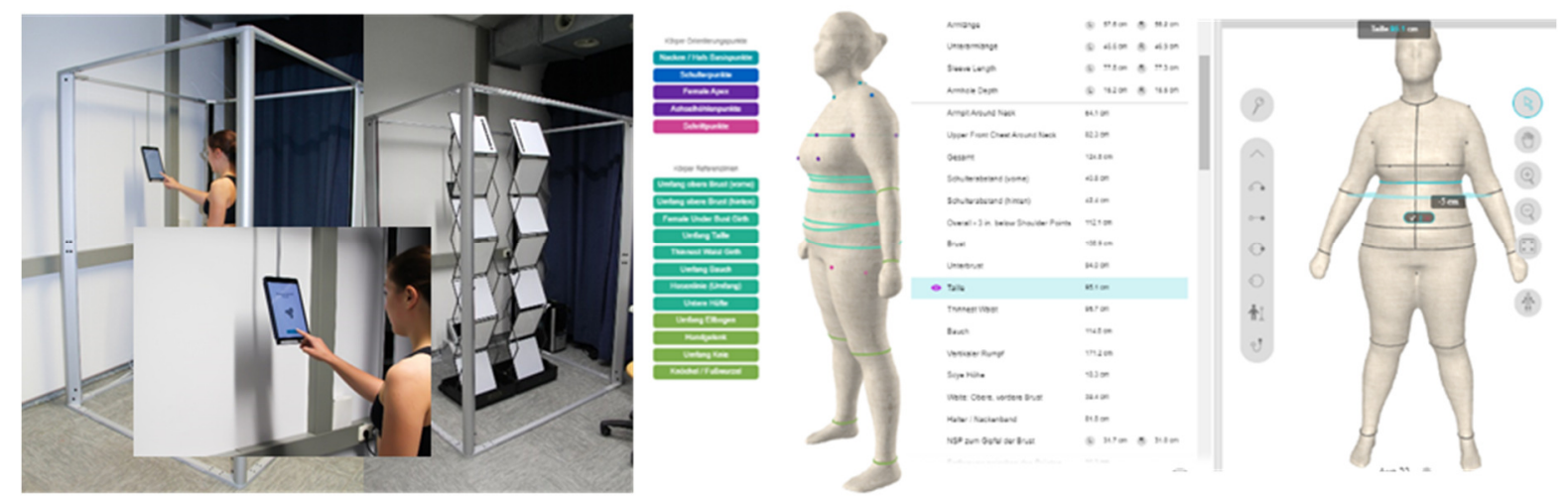

Figure 3: TG3D Scanatic 360 Body Scanner and evaluation software

As shown in table 2 the usage and comparison of different scanning technologies results in different body measurements, strongly depending from the particular algorithm used. Especially for bodies not representing the common expression of body shapes, the localization of the measurements has to be controlled and adapted if necessary to get reliable results. In table two the measurements have been taken directly from the individual scanning software, afterwards subjected to a validation process and compared also to manually taken measurements from experts and the scanning subject and our quick size function. The manually taken measurements from several experts have been taken finally as reference.

For female F1 the taken measurements are nearby. In general the scanning results for F1 differ not extremely when comparing to the simple quick size function- also due to representing an average body shape by scanning subject female $\mathrm{F} 1$. Other scanning subjects deviating stronger from average are showing easily scan data variation in range of $4-8 \mathrm{~cm}$ and therefore reaching 1-2 grading step values. These deviation are necessary to examine with regard to finished measurements of the product and a personal feeling of individual subjects when selecting their "personal" size.

\begin{tabular}{|c|c|c|c|c|c|c|c|}
\hline \multicolumn{8}{|c|}{ Female F1 } \\
\hline body measurement $[\mathrm{cm}]$ & HME & HMS & QSF & 1 & $\mathrm{~L}$ & K12 & K1 \\
\hline bust (uBr) & 106 & 105 & 107,8 & 106,2 & 106,6 & 109,1 & 104,1 \\
\hline waist (uTa) & 89 & 88 & 91,9 & 90,8 & 92,8 & 94,1 & 94,2 \\
\hline hip (uGe) & 116 & 114 & 113,4 & 116,9 & 116,8 & 118 & 117,1 \\
\hline
\end{tabular}

Table 2: Comparison female F1: HME (hand measurement expert), HMS (hand measurement subject), QSF (quick size function), I (TG3D), L (avalution Laser), K12 (avalution Kinect), K1 (Styku Kinect)

Anyway, the quick size function is an easy to use simple tool for size prediction in a first approach - if only little information are given such as age, body height and body weight but a recommendation should be given to a potential customer anyway. 


\subsection{Front/Side View Algorithm}

Often in E-commerce customers can upload two photos representing their bodies in front and side view. Extracting measurements from these pictures and calculating body volumes according to human being body models, offers on one side the possibility of calculating body measurements for size prediction and on the other generating a 3D avatar matching the individual shape.

Front and side pictures from sample sets have been analyzed by using Profile Fit Pattern (PFP Suite Grafis) and by developing algorithm based on advanced elliptical models from Ramanujan resulting in body measurement prediction and avatar built.

The most critical point is the quality of pictures created by customers themselves and not controlled under laboratory conditions and taken by experts. Apart from the fact that not all people are willing to upload private pictures- especially in higher age groups- although privacy is guaranteed by the company/platform- the conditions like light setting, distance and angle to camera position, background and finally the definition of tight fitted underwear do have a non-negligible impact on the following steps when trying to extract body information from two photos.

In Fig. 4 the elliptical approach of cut sections along body height is shown. By rating and categorizing the two axis of the ellipse according to ratio and body height location, the calculation done with our model show acceptable results for individual test specimens within tolerances of max. one grading step.
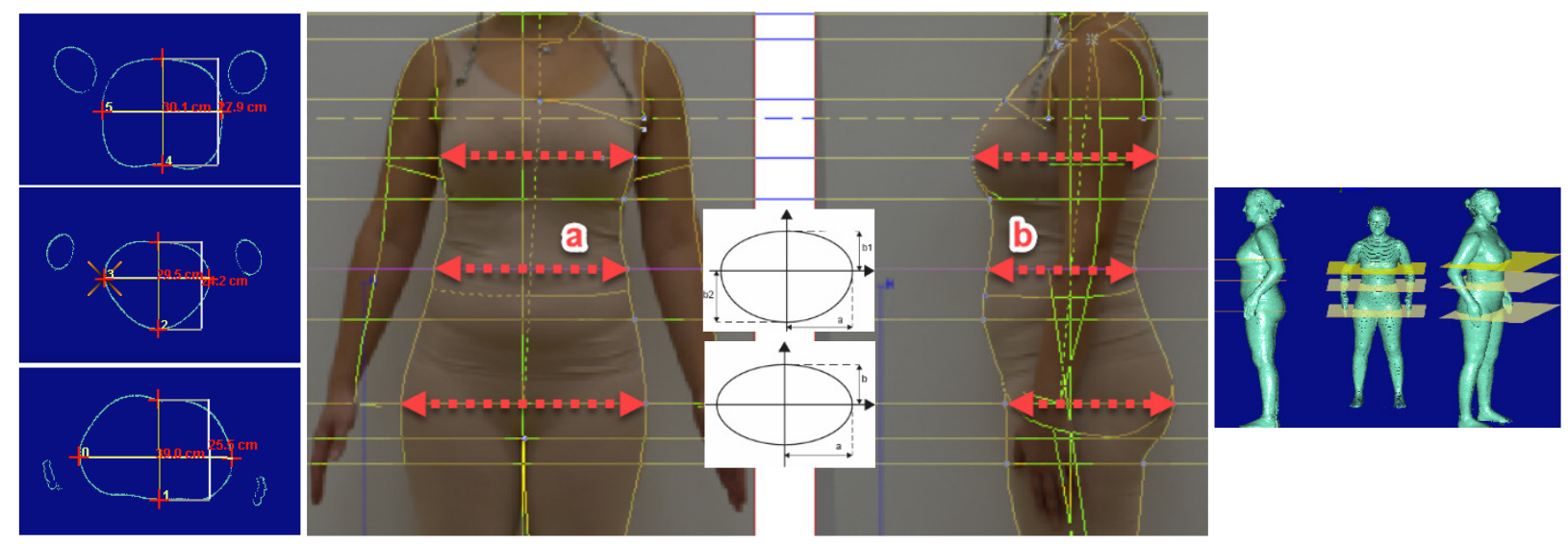

Figure 4: Elliptical model approach for body measurement calculation; female 4

In table 3 the results for female $\mathrm{F} 2$ are presented in comparison to the other methods like hand measurement, quick size function and scanning technology. As shown, the calculations by using the elliptical model developed in house are also nearby the measurements taken by expert and therefore taken as reference, acceptable for an industrial process.

\begin{tabular}{|c|c|c|c|c|c|c|c|c|c|}
\hline \multicolumn{10}{|c|}{ Female F2 } \\
\hline body measurement [cm] & HME & HMS & QSF & 1 & $\mathrm{~L}$ & K12 & K1 & PFP & $E$ \\
\hline bust (uBr) & 109 & 105,5 & 111,5 & 108 & 108,1 & 115,3 & 104,6 & 112,6 & 107,7 \\
\hline waist (uTa) & 97 & 100 & 97,1 & 99,5 & 105 & 107,2 & 102,9 & 101,9 & 101,8 \\
\hline hip (uGe) & 117 & 116 & 112,7 & 117,4 & 116,6 & 118,7 & 116,6 & 116,8 & 119 \\
\hline
\end{tabular}

Table 3: Comparison female F2: HME (hand measurement expert), HMS (hand measurement subject), QSF (quick size function), I (TG3D), L (avalution Laser), K12 (avalution Kinect), K1 (Styku Kinect), PFP (Profile Fit Pattern), E (elliptical Model)

Whereas scanning technology results also in avatar creation, quick size function and our elliptical model require this step additionally. In both cases we are referring to our avatar population tool, based on human model (VirLab; avalution [4]), to output an avatar, useful for a 3D fitting process. Apart from size prediction based on the technologies presented, the avatar shown in E-shopping application to customers is a highly critical discussed topic. Apart from simulation restriction especially the representation of the real body on one side and the design of the avatar on the other is positioned in a field of fashion and commercial topics: Finally, the customer should be convinced and make a positive buying decision and not be disappointed when "opening the parcel" or try on the ordered clothing. 


\section{Avatar Acceptance}

Generating avatars for a virtual shopping experience is difficult due to realism and acceptance.[6] On one side the avatar should represent the body to show the selected size in a virtual preview best possible. On the other side people have a personal idea and vision when it comes to fashion products. Though often in reality of promotion the mannequins do not represent really the target group, these mannequins are trying to "seduce" the customer in a world of illusion

The fact that the presented garment will look not the same when worn by the customer is not as much important as the feeling of the customer and the association given in mind.

Therefore, when it comes to individual body measurements, a perfect scan showing all the details of a body is not well accepted compared to avatars with smoothed surface and not showing the last little detail.

Avatar design- especially head, face, arms, hands and feet- is also a complex topic and compared to avatars for product development accepted by design and the technical department at least as much important as for customers at POS.

Realism of avatars is in contrast to brand language, possibilities of rendering and comes with surface and background colors combined with fabric pattern and color management.

In Fig. 5 different avatar levels are shown.

By questioning the subjects involved into this research and extending to a broader audience the general outcome was an avatar representing the basic shape of the customer in body shape, neutral clean surface, giving an impression of a personal feeling and anyway no virtual twin.

Only the presentation of the brand idea- not customer fitting- was favored in corresponding acceptance of a high-end rendered avatar.

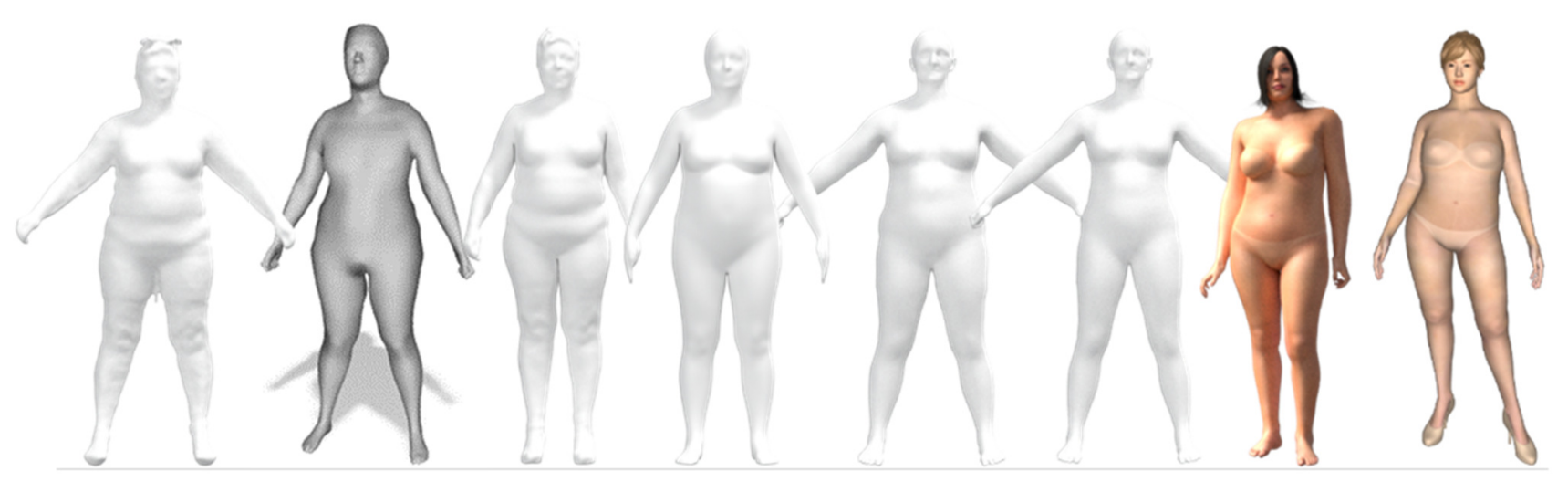

Figure 5: Avatar design levels for E-shopping and acceptance by customer, Female F4; Styku-TG3D-VitusAvatartool-QSF-App avatar-vstitcher model-TG3D model

\section{Target Avatar Population}

Based on the feedback we have decided to create an avatar population representing base size avatars brand related (female size 34- 48, male size 46-54) and for each of them a target group modification for better presentation of individual body shapes.

The complete avatar population is available for our quick size function and our elliptical model by assigning the best matching avatar to customer profile after extracting at least body height, bust, waist and hip measurements. Avatar design was also aligned to feedback of a questionnaire and therefore kept simple in surface color and hair design.

Variations of body height, waist and hip measurements by defining bust measurement as primary measurement have been tested to investigate the overall visual expression and rating by customer. Respecting pattern design and virtual fabric parameters (physical and visual), it was possible to reduce the avatar population due to negligible visibility of fit quality. 
The reduction of the avatar population keeps down also the simulation preparation process - in most cases the simulation still cannot be done in real time after customer selection due to simulation challenges of complex garments and outfits or by running via batch process with adjusting the simulations if true to life simulation results are afforded. Thus, we use a "rigging process" to speed up the simulation process after avatar exchange.
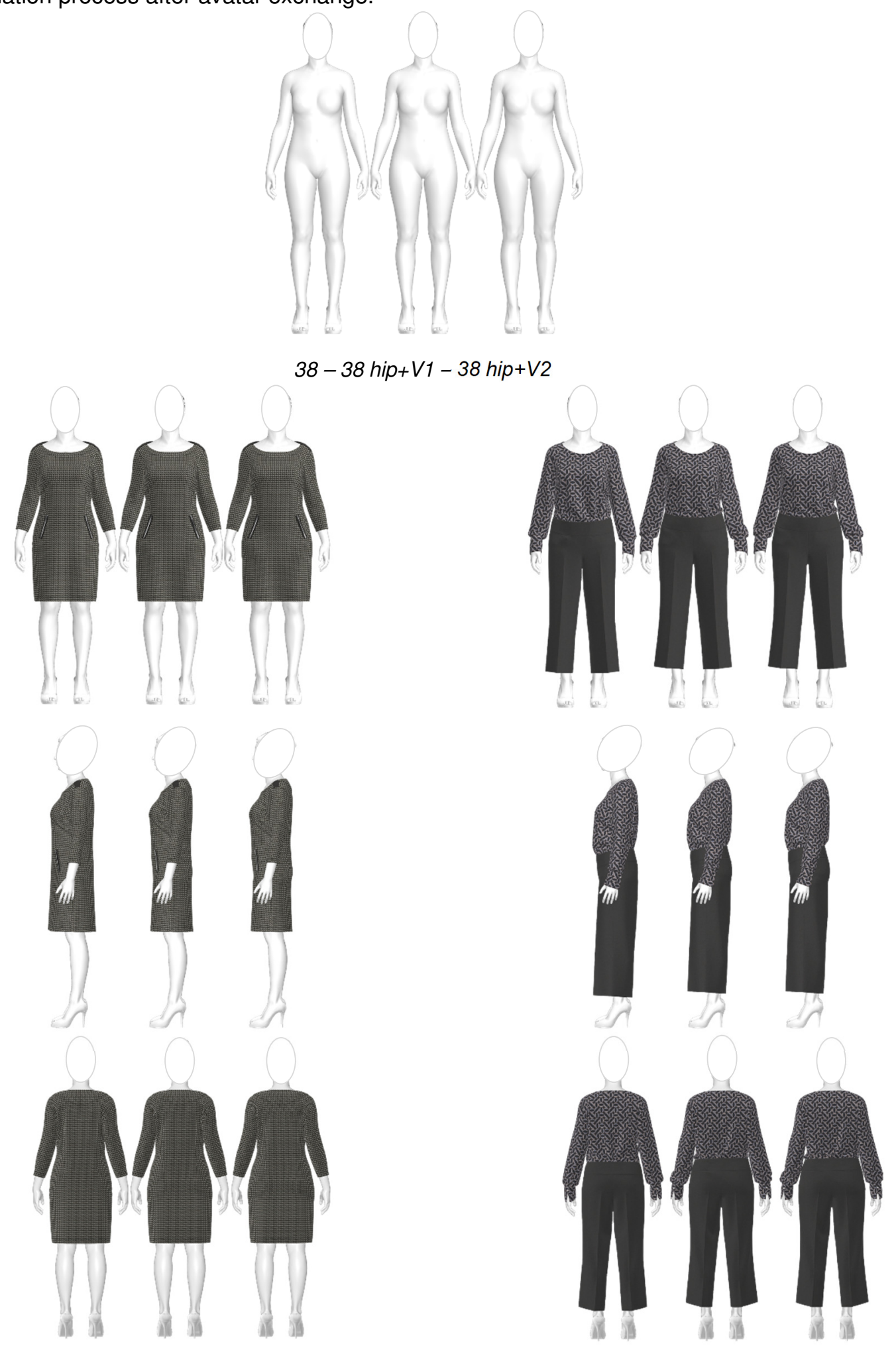

Figure 6: Extract of avatar population size 38 - $38 \mathrm{hip}+V 1(4 \mathrm{~cm})-38 \mathrm{hip}+\mathrm{V} 2(8 \mathrm{~cm})$; body height fixed; bust fixed, waist fixed; preselection for representing customer profile, blouse 38; trousers 38, dress 38 
Another critical point is also detectable: In figure 6 the impact of fabric maps becomes directly visible. These maps have been created by using TG3D Nuno Fabric Scanner and Vizoo system for comparison. Due to mapped color and pattern files most of the little details concerning stretch and pleats do not become visible anymore- especially after uploading the simulation as viewer files to our test platforms in E-Shopping.

Simulation are done in various systems like TG3D,CLO, Vstitcher, vidya..- results shown in fig. 6 and fig. 7 are created with DCSuite from TG3D; results shown in all simulation systems almost the same. Figure 7 shows simulation examples in plain white color- in comparison to figure 6 . Now a lot of more details become visible. Also fabric from Nuno fabric scanning and after assigning in DCSuite is shown.

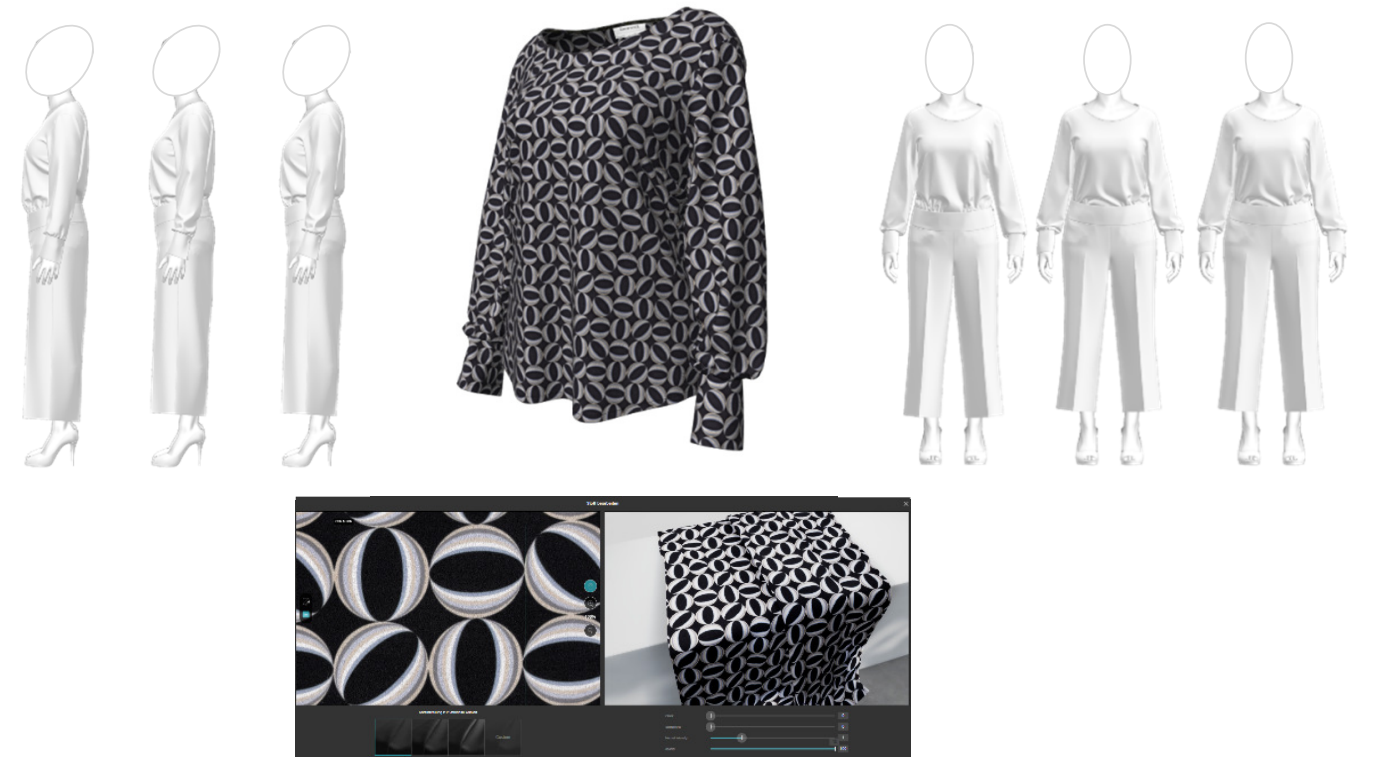

Figure 7: Extract of avatar population size 38-38 hip+V1-38 hip+V2; size 38 plain white; $D C$ simulation and Nuno fabric scanning TG3D

\section{Size Prediction}

Apart from avatar design, size prediction is one of the most critical process steps within E-shopping. If only information are given coming from quick size function (QSF) or via our elliptical model (E) but also from body scanning process, size recommendation can only be given by comparing the calculated body measurements with finish measurement of the products- usually available from product development or quality check. The first challenge is to combine body measurement location with finish measurement location of clothing- in most cases not matching. (e.g. bust measurement body versus $1 / 2$ bust measurement relocated to armhole bottom of the product)

Also the fact that different garments have different ease added on to certain body positions depending from brand, design, fabric complicate this process step.

But the most critical point is the personal feeling and fit understanding of individuals when it comes to clothing. In our size prediction model we have detected, that most of the females have personally selected one or more sizes smaller recommended by calculation. This can also be effected by so called "adulate sizing" of companies to communicate the feeling of well-being when wearing a smaller size. The personal decision can differ from product to product (jacket, skirt, dress) and is also affected by fabric properties. [7]

Thus we conclude that additional personal information are the most important information necessary for a personal size prediction and can be finally more important than single data created by tools mentioned. These information can be obtained by collecting customer data concerning already bought products from the same company, from competitors, from return reports, and also additional information shaping a "personal" model of people never seen or met before.

Figure 8 shows females in different sizes, assigned via size prediction and via personal feeling. The challenge and the limits of size prediction by using only calculations from obtained body measurements does not fulfill the requirements when it comes to personal feeling and decision of customer and can only be sharpened by collecting more information to create a virtual personal profile. 
The general size prediction was defined by rating all three measurements and taken the highest one in a first approach to get the bottle neck. By comparing the different methods and extracting measurements by automatism it is obviously visible, that all of them are showing results close by but usually too high. Personal feeling of all subjects was in general at least one size smaller than the general size prediction and therefore rated as "adulate sizing", created by company.

\begin{tabular}{|c|c|c|c|c|}
\hline \multirow{2}{*}{ Method } & \multicolumn{3}{|c|}{ Female F1 } & General Size \\
\cline { 2 - 4 } & $\mathrm{uBr}$ & $\mathrm{uTa}$ & $\mathrm{uGe}$ & \\
\hline $\mathrm{PFP}$ & 46 & 44 & 44 & 46 \\
\hline $\mathrm{I}$ & 44 & 46 & 46 & 46 \\
\hline QSF & 46 & 46 & 44 & 46 \\
\hline E & 44 & 46 & 46 & 46 \\
\hline
\end{tabular}

\begin{tabular}{|c|c|c|c|c|}
\hline \multirow{2}{*}{ Method } & \multicolumn{3}{|c|}{ Female F3 } & General Size \\
\cline { 2 - 4 } & $\mathrm{uBr}$ & $\mathrm{uTa}$ & $\mathrm{uGe}$ & \\
\hline PFP & 42 & 42 & 40 & 42 \\
\hline $\mathrm{I}$ & 40 & 42 & 40 & 42 \\
\hline QSF & 42 & 42 & 40 & 42 \\
\hline E & 40 & 42 & 40 & 42 \\
\hline
\end{tabular}

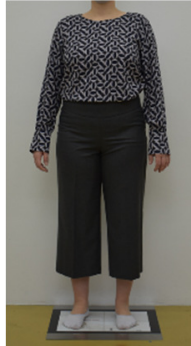

42

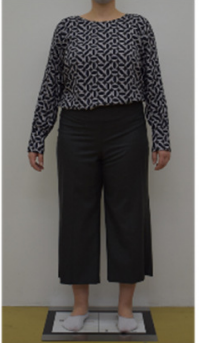

44

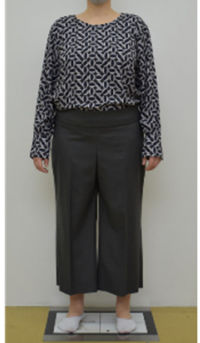

46

blouse: rec. 46 / pers. 42 ; trousers: rec. 46 / per. 44

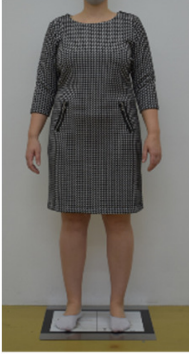

42

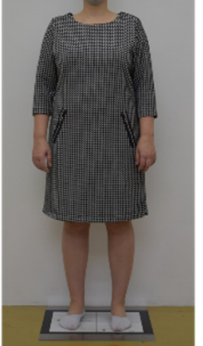

44

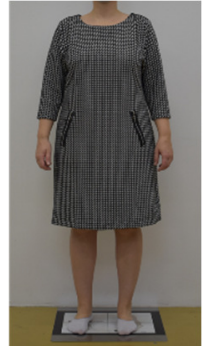

46

dress: rec. $46 /$ pers. 44

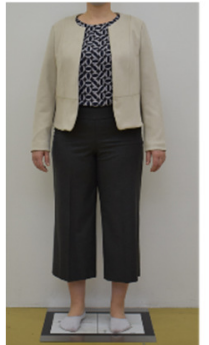

42

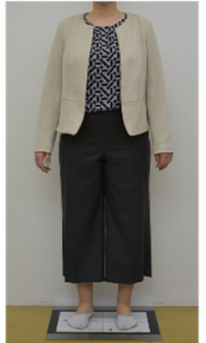

44

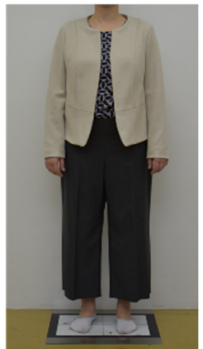

46

jacket: rec. 46 / pers. 44

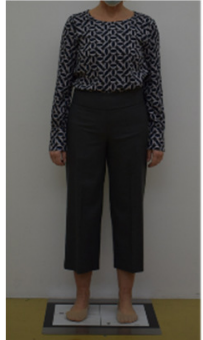

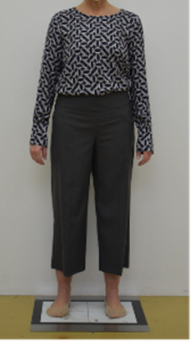

38

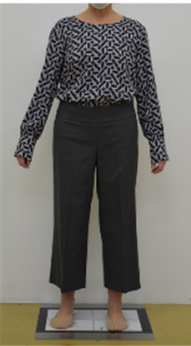

40 blouse: rec. 42 / pers. 38 ; trousers: rec. 42 / per. 38

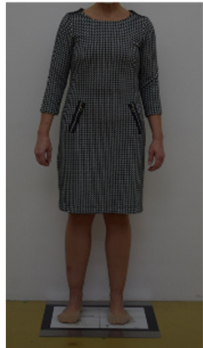

36

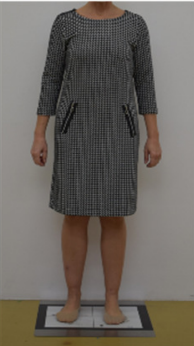

38

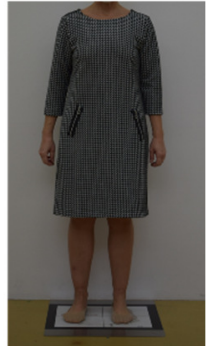

40 dress: rec. 42 / pers. 38

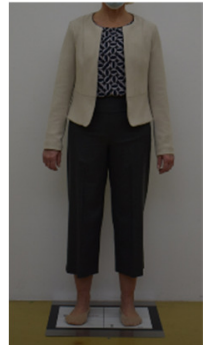

36

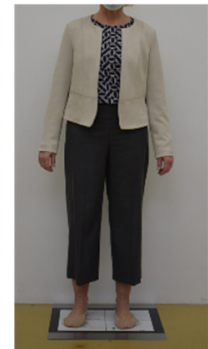

38

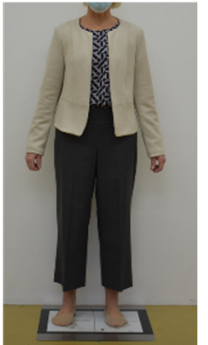

40 jacket: rec. 42 / pers. 38

Figure 8: Real fitting with different sizes; size prediction (rec.) and personal size feeling (pers.) 
Also the fact that people are used to a personal size and "always" only accept this size- although one size plus would be much better, was often detected. So female F3 is used to be a size 38 customer in her personal feeling.

\section{E-Shopping Concept}

In an E-shopping concept the individual measurements are captured by quick size function or if available and accepted by the customer by uploading two photos (front and side view) to the platform. The resulting size prediction is called "I love my size" and offers a preselected avatar available for a virtual fit session in the "closet" area created in the shop. If no personal data are given, no size prediction is available and finally the virtual fitting is only shown in base size on base avatar to offer this feature anyway.

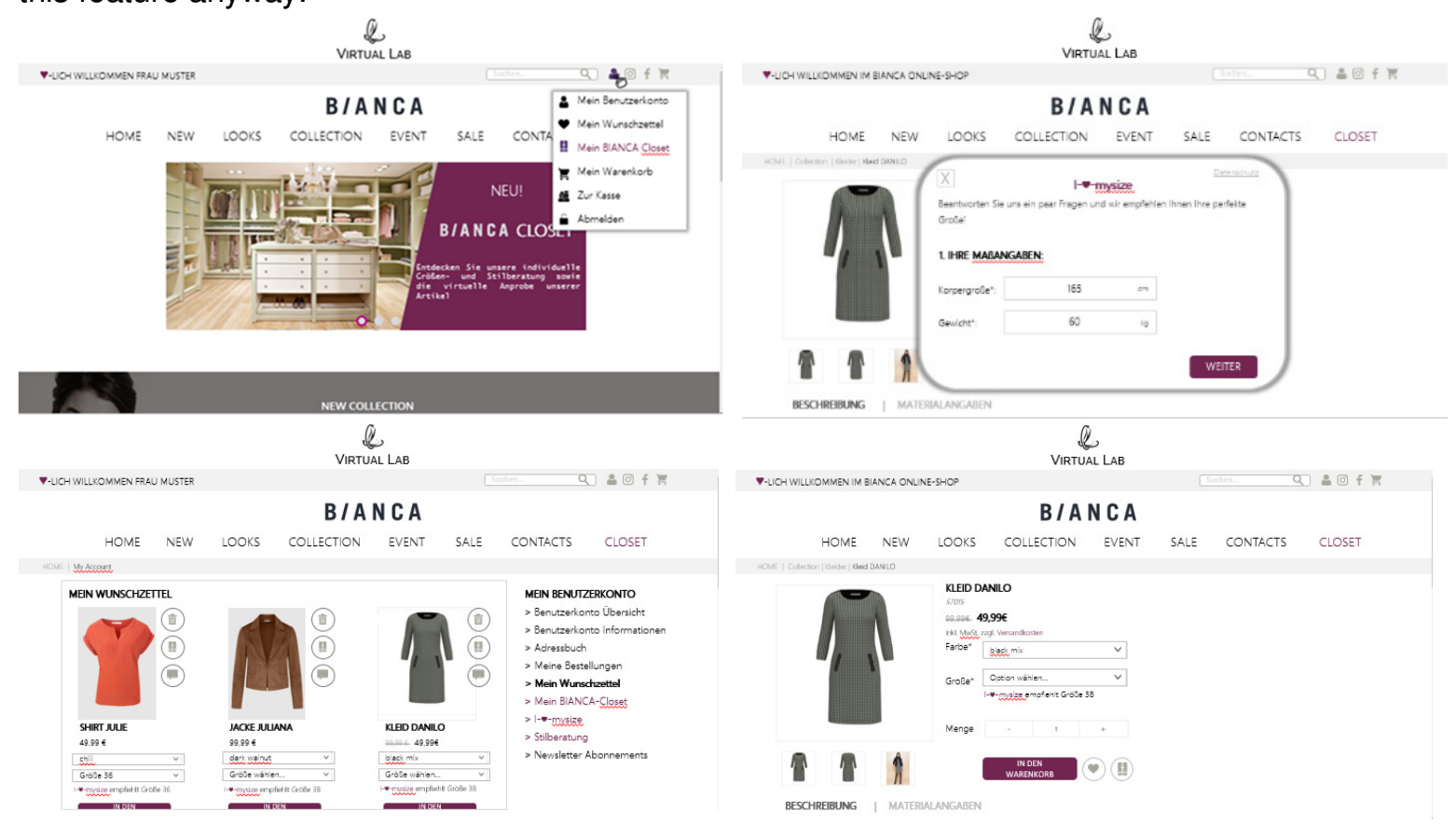

Figure 8: Product selection and "I love my size" function in E-Shopping concept; Virlab\&Bianca

\subsection{The Virtual Closet}

Customers can float through the collections and save preselected garments in the closet area. In this area the preselected garment toolbar is extended by a recommendation toolbar and a so called purchase toolbar, where products already purchased are shown, and are available to combine with the new products in the mirror windows and by activating also simulated on the avatar for showing a virtual fit by using a 3D viewer.

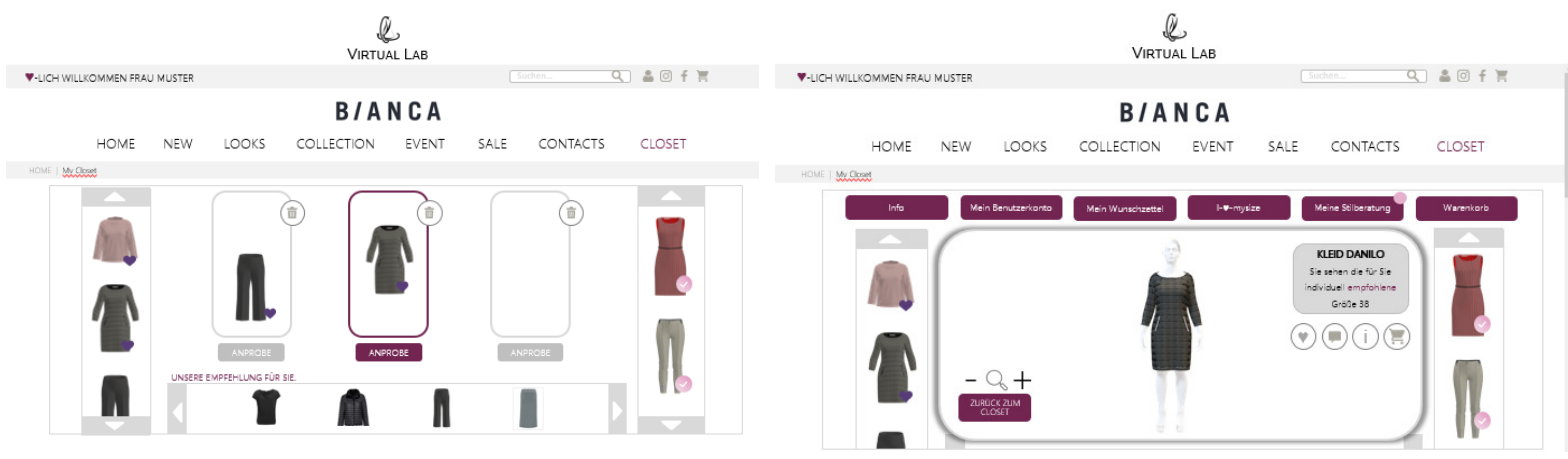

Figure 9: E-commerce closet, fitting room and "love my size" function; VirLab for Bianca company 


\subsection{Online APP Store}

Additionally a virtual shopping app using TG3D cloudzet store app /web plugin was created by individualizing given templates and uploading the simulation results created with a so called Bric function (block system) in DC Suite of TG3D 2D3D cad program. [9]

Compared to the complex shopping concept developed and programmed before, this tool offers a quick solution for creating an own shop concept store based on 3D simulation and 3D avatars combined with size prediction and the possibility for MtM (made to measure) extension.
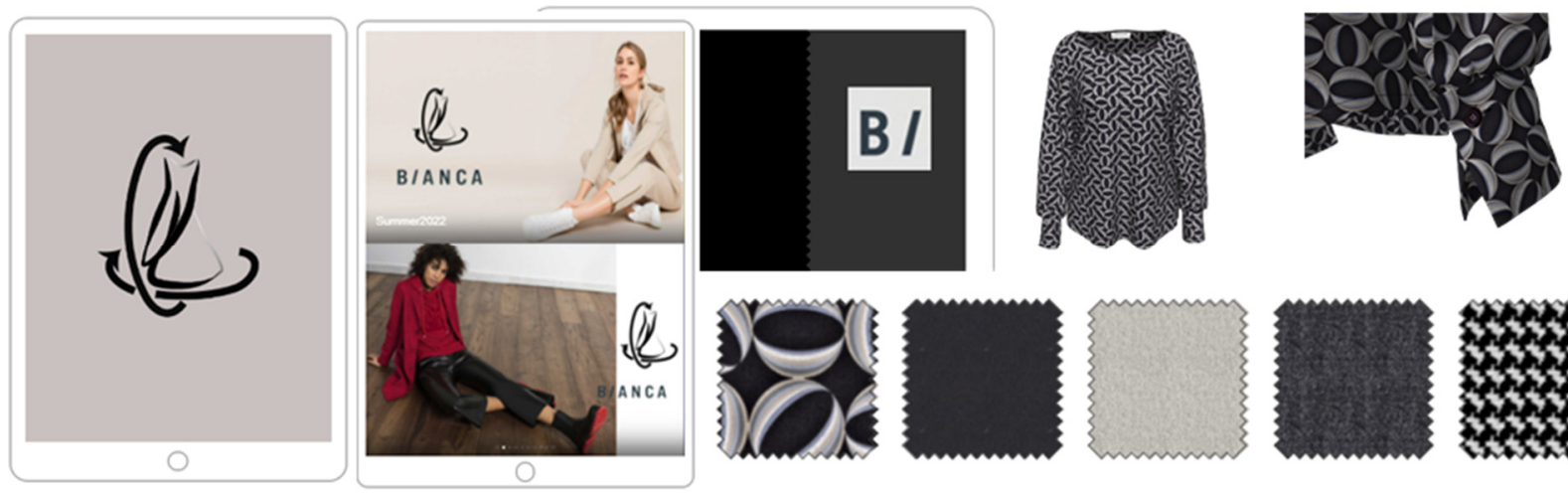

Figure 9: E-commerce Shopping App; VirLab based on TG3D store concept

Comparing both concepts, it is obviously shown, that concept 1-building up the new features in an already existing online shop concept is time and cost intense, but offers the freedom of implementing the tools in a much more customized manner. Taking already existing solutions and templates such as offered by TG3D are an effective way to create quickly a new shop concept based on features such as body scanning, fabric scanning, 2D/3D Cad, by saving time and costs but offering anyway a very customer friendly solution and atmosphere aimed for acquiring new customers in E-commerce.

By using and combining all parts of a complex but streamed digital solution, especially valuable time can be saved compared to a mix of single solutions with steady import and export challenges- we call this "program hopping". Thus especially for a start-up, the small business sector, but also for companies not able to build up a complete mixed process with high end solutions from scratch, this is a proper solution in a fast turning business to benefit from a virtual product development process up to point of sale with manageable invest.

\section{High End Brand Avatar}

Discussing the avatar design and the simulation quality, rendering becomes more and more important to fulfill the requirements of a real true to live experience for the customer. Especially if highlight pictures usually created in photo studios or in nature are needed. Apart from time and cost efficiency we have investigated the possibilities of rendering by following import- export paths from DAZ Studio and cinema to various 3D cad system and back usually via Blender bridge. The avatar created in DAZ Studio undergo a made to measure process to adjust them according our target population for E-commerce. This avatar population is represented by a single adjustable avatar called "Mylene" and imported in static pose or as animation file to our 3D systems. After running the simulation and if necessary calculating the simulation frames, we pave the way back to the original place and start the rendering process.

Up to now this process is time and cost intense- therefore we are investigating an automatically running process adaptable to our $3 \mathrm{D}$ simulation programs for clothing. [8]

Although all of these 3D programs are offering a kind of rendering and for most application in the product development process sufficient, the results usually do not match the requirements coming from promotion and sales.

Realizing that most people do not want to see a perfect twin of themselves instead of an inspiring avatar, we are focusing on a virtual mannequin representing the vision of a companies' designer and the mood transferred with their collection to present the products also for eye catching at the beginning of the shopping experience. 


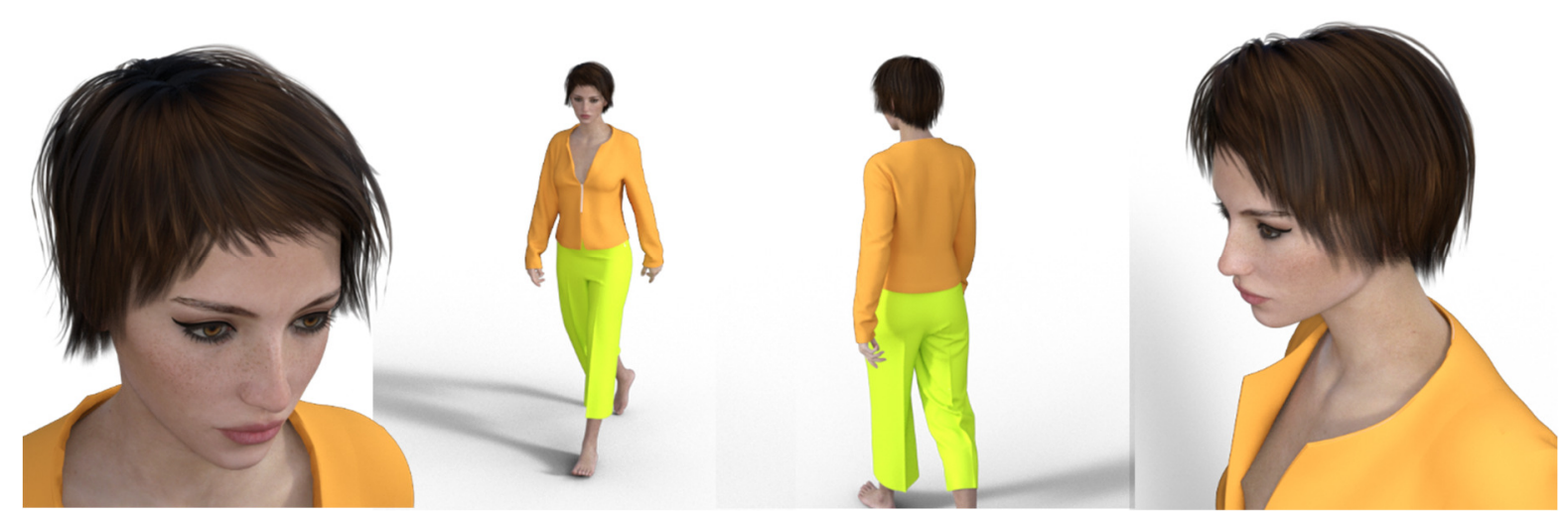

Figure 10: VirLab model "Mylene", size adapted and rendered in DAZ Studio after reimport cloth simulation

\section{Summary}

E-commerce and E-shopping are a versatile "playground" hunting for customers and finally sales volume. A lot of questions and challenges are popping up when it comes to size prediction, representing avatars, simulation and rendering and most of them are directly superimposed by a subjective feeling of the customer, difficult to assess by two photos and a few questions only.

By investigating different method for firstly get a body measurement information and secondly deriving a size prediction all of them have their limitations: Personal feeling and shopping preference/history are more value than predicting a size based on mostly doubtful body measurements, coming from selfies or questioning body information.

Avatar design is also a critical point: In our research and within the years of body scanning and avatar creation in our lab, the majority of people has strictly denied to see and accept a twin of their own, preferring an attractive virtual mannequin, the same as in reality of fashion business. By creating neutral avatars representing the customer in a virtual fitting room and a branded avatar like our "Mylene", representing a brands model, both "parties" are benefitting in this process: the company and the customer.

In the virtual fitting process we have seen, that all of the systems have still their limits due to limited physical fabric parameters, simulation settings, avatar incompressibility and the gap between the virtual manufacturing process and the reality in industry. Simulation of complex garments and outfits from scratch without "reworking" and "guiding" the simulation" is still a big challenge when it comes to simulations on individual customer avatars and the question arise if this is really wanted respectively what is really visible.

Finally the building of an Ecommerce platform and offering all the information in a way that potential customers are attracted and satisfied and maybe increase their loyalty is a subtle and fragile composition on tools provided to them when it comes to sizing and fit. The missing product "touch" and fabric "feeling", given in reality and with a huge impact on shopping behavior is compulsory necessary to involve and by using artificial intelligence methods the personal customer profile should provide more information for product and size recommendation in virtual shopping- still not really knowing the customer.

\section{Outlook}

Avatar design, sizing and fit, 3D simulation and rendering are necessary disciplines for a high-end virtual shopping experience. The fact, that each of them is a wide field and all of them have to come together is a challenging task- especially in the world of fashion with lots of uncertain personal feelings difficult to catch and rate and maybe quickly different tomorrow and therefore hard to deliver.

Anyway E-commerce and E-shopping are useful tools to offer clothing to a broad market. The combination of all the given disciplines in a fluent way already coming from a well-organized virtual product development process lifted up to a complete virtual process with beginning at design state and downloaded not before production, communicating completely digitally, also pave the path for a successfully sustainable digital shopping experience in the near future. 


\section{References}

1. Ernst, M.; Christophersen, A.; Botzenhardt, U.: "Efficient Virtual Garment Fit Evaluation Infrastructure based on Synthetic Avatar Target Customer Groups for MtM Application", in Proc. of 3DBODY.TECH 2018- 9 $^{\text {th }}$ Int. Conf. and Exh. On 3D Body Scanning and Processing Technologies, Lugano, Switzerland, 16-17 October 2018

2. Magenant-Thalmann, N.; Thalmann, D.: "Handbook of Virtual Humans"; 2004 John Wiley \& Sons, Ltd ISBN:9780470023167

3. Yuzhao L.; Yuhan L.; Shihui X.: "Comparing VR- and AR-Based Try-On Systems Using Personalized Avatars" ; Electronics 2 020, 9, 1814; doi:10.3390/electronics9111814

4. Rissiek, A.: 3D Bodyscanning Reihenmessungen und Avatargenerierung als Türöffner für die 3D Produktentwicklung von Bekleidung; DTB Tagung 06-07 July 2021, Düsseldorf

5. Daanen, H.; Psikuta, A.: Automation in Garment Manufacturing 2018, p. 237-252, Elsevier Woodhead Publising

6. Ridgway, J. L.: "Before and After Avatar Exposure: The Impact of Body Scanning Technology on Body Satisfaction, Mood, and Appearance Management"; Clothing and Textiles Research Journal 2018, Vol. 36 (2) 91-103

7. Gersak, J.: Complexe fabric deformations and clothing modelling in 3D, Lambert Academic Publishing; ISBN: 978-3-659-32809-1

8. Magenant-Thalmann, N.: Modeling and Simulating Bodies and Garments, Springer 2010, ISBN: 978-1-84996-262-9

9. KO, Heyong-Seok: Digital Clothing; Seoul, South Corea 2015, ISBN: 978-89-363-1449-1 